\title{
Off-forward gluonometry.
}

\author{
A.V. Belitsky ${ }^{a}$, D. Müller ${ }^{b}$ \\ ${ }^{a}$ C.N. Yang Institute for Theoretical Physics \\ State University of New York at Stony Brook \\ NY 11794-3840, Stony Brook, USA \\ ${ }^{b}$ Institut für Theoretische Physik, Universität Regensburg \\ D-93040 Regensburg, Germany
}

\begin{abstract}
We explore the deeply virtual Compton scattering process at NLO with the emphasis on the measurement of the tensor gluon skewed distributions. We recalculate the one-loop coefficient function and give two-loop results for anomalous dimensions and exclusive evolution kernels required for studying the logarithmic scaling violation. We evaluate weighted cross sections which give direct access to these functions on experiment.
\end{abstract}

Keywords: deeply virtual Compton scattering, tensor gluon operator, NLO calculations

PACS numbers: 11.10.Hi, 12.38.Bx, 13.60.Fz 


\section{Introduction.}

The studies of the gluonic content of hadrons are complicated by the fact that there exists no probes which couples directly to it. The only way for electroweak bosons to fuse with coloured gluons is via quark loops. Obviously this is an effect suppressed in the QCD coupling constant on the background of dominating tree level quark contributions. Gluon distributions mix logarithmically with quark ones and although they can affect the shape of the latter in a significant way it is still extremely complicated to disentangle them in perturbative evolution. An opportunity to measure gluons directly could appear provided there is a selection rule which forbids quarks to enter as a leading effect in the cross section.

At leading twist-two level there are three non-local light-ray operators bilinear in gluon fields. E.g. in the light-cone gauge where only transversely polarized vector fields do propagate, we have the following projections for the Lorentz structure of the composite operator $B_{\mu}\left(x_{1}\right) B_{\nu}\left(x_{2}\right) g_{\mu \mu^{\prime}}^{\perp} g_{\nu \nu^{\prime}}^{\perp}$

$$
g_{\mu \mu^{\prime}}^{\perp} g_{\nu \nu^{\prime}}^{\perp}=\frac{1}{2} g_{\mu \nu}^{\perp} g_{\mu^{\prime} \nu^{\prime}}^{\perp}+\frac{1}{2} \epsilon_{\mu \nu}^{\perp} \epsilon_{\mu^{\prime} \nu^{\prime}}^{\perp}+\tau_{\mu \nu ; \rho \sigma}^{\perp} \tau_{\mu^{\prime} \nu^{\prime} ; \rho \sigma}^{\perp},
$$

where $g_{\mu \nu}^{\perp}=g_{\mu \nu}-n_{\mu} n_{\nu}^{\star}-n_{\mu}^{\star} n_{\nu}, \epsilon_{\mu \nu}^{\perp}=\epsilon_{\mu \nu \rho \sigma} n_{\rho}^{\star} n_{\sigma}$ and $\tau_{\mu \nu ; \rho \sigma}^{\perp}=\frac{1}{2}\left(g_{\mu \rho}^{\perp} g_{\nu \sigma}^{\perp}+g_{\mu \sigma}^{\perp} g_{\nu \rho}^{\perp}-g_{\mu \nu}^{\perp} g_{\rho \sigma}^{\perp}\right)$. Here $n_{\mu}$ and $n_{\nu}^{\star}$ are two light-cone vectors such that $n^{2}=n^{\star 2}=0$ and $n n^{\star}=1$. Eq. (1) corresponds to the Clebsch-Gordon decomposition of the direct product of the two vector representations of the Lorentz group円 $\left(\frac{1}{2}, \frac{1}{2}\right) \otimes\left(\frac{1}{2}, \frac{1}{2}\right)=(0,0) \oplus((1,0) \oplus(0,1)) \oplus(1,1)$. The first two tensors stand for vector and axial sectors and they contribute to the conventional deep inelastic scattering (DIS) process on spin- $\frac{1}{2}$ hadrons. The last one cannot appear there since it requires the photon helicity to be flipped by two units. However, it definitely can appear in scattering on spin $J \geq 1$ targets as was emphasized in Ref. [1]. Since these operators belong to the spin-2 representation $(1,1)$ of the Lorentz group, they cannot mix with quark operators. Therefore they can serve as a clean probe of the gluonic content in hadrons not contaminated by other effects. Recently this issue was discussed in the context of current fragmentation in DIS [2] and in the production of two pions in $\gamma \gamma$ fusion [3]. Nevertheless this operator can appear in the Compton scattering on the nucleon provided it is sandwiched between states with different momenta as was pointed out [4] and elaborated in Ref. [5]. The corresponding kinematics of the process is known as deeply virtual Compton scattering (DVCS) [6, 7, 8]. In this note we address the issue of the tensor gluon skewed parton distributions (SPD) in DVCS in great detail. We recalculate the one-loop coefficient function, and present two-loop anomalous dimensions and exclusive evolution kernels for this sector. We give an explicit prediction for weighted cross sections which can be used to extract directly the tensor gluon SPD from experimental data.

\footnotetext{
${ }^{1}$ Recall that the representations of the Lorentz group $L_{+}^{\uparrow}=S O(3,1)=S O(4, \mathbf{C})_{\downarrow R} \approx(S L(2, \mathbf{C}) \otimes S L(2, \mathbf{C}))_{\downarrow R}$ are labeled by a pair $\left(j_{1}, j_{2}\right)$ which are eigenvalues $j_{i}\left(j_{i}+1\right)$ of the $S L(2)$ Casimir operators $\hat{\boldsymbol{J}}_{i}^{2}$.
} 

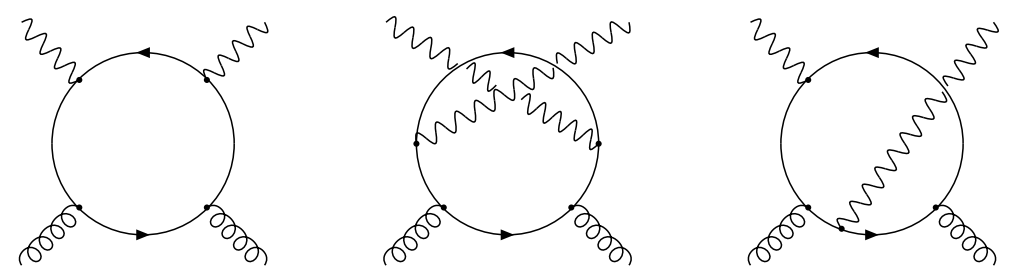

Figure 1: One-loop diagrams for the coefficient function.

\section{$2 \quad$ Leading twist amplitude.}

The hadronic part of the deeply virtual Compton scattering amplitude is defined by the off-forward matrix element of the correlator of two electro-magnetic currents sandwiched between states with unequal momenta

$$
T_{\mu \nu}\left(q, P_{1}, P_{2}\right)=i \int d x e^{i x \cdot q}\left\langle P_{2}\left|T j_{\mu}(x / 2) j_{\nu}(-x / 2)\right| P_{1}\right\rangle
$$

where $q=\left(q_{1}+q_{2}\right) / 2$ (and the index $\mu$ refers to the outgoing real photon with momentum $q_{2}$ ) and $P_{1}\left(P_{2}\right)$ is the momentum of incoming (outgoing) nucleon. The leading contribution of the light-ray tensor gluon operator

$$
{ }^{G} \mathcal{O}_{\mu \nu}^{T}\left(\kappa_{1}, \kappa_{2}\right)=G_{+\rho}\left(\kappa_{2} n\right) \tau_{\mu \nu ; \rho \sigma}^{\perp} \Phi\left[\kappa_{2} n, \kappa_{1} n\right] G_{\sigma+}\left(\kappa_{1} n\right)
$$

whose off-forwards matrix element is parametrized via two SPDs [5]

$$
\begin{gathered}
G_{\mu \nu}^{T}\left(t, \eta, \Delta^{2}\right) \equiv 4 P_{+}^{-1} \int \frac{d \kappa}{2 \pi} e^{i \kappa t P_{+}}\left\langle\left. P_{2}\right|^{G} \mathcal{O}_{\mu \nu}^{T}(\kappa,-\kappa) \mid P_{1}\right\rangle=H_{G}^{T}\left(t, \eta, \Delta^{2}\right) \frac{\tau_{\mu \nu ; \alpha \beta}^{\perp}}{2 M} \frac{\Delta_{\alpha} q_{\gamma}}{P \cdot q} \bar{U}\left(P_{2}\right) i \sigma_{\gamma \beta} U\left(P_{1}\right) \\
+E_{G}^{T}\left(t, \eta, \Delta^{2}\right) \frac{\tau_{\mu \nu ; \alpha \beta}^{\perp}}{4 M^{2}} \Delta_{\alpha} \bar{U}\left(P_{2}\right)\left(\frac{\Delta_{\beta} \not q}{P \cdot q}-\eta \gamma_{\beta}\right) U\left(P_{1}\right)
\end{gathered}
$$

into the operator product expansion of currents (2) appear at one-loop order (see Fig. 巴). The traceless symmetric projector $\tau^{\perp}$ in Eq. (四) possesses the properties $\tau_{\mu \nu ; \rho \sigma}^{\perp} \tau_{\mu \nu ; \rho^{\prime} \sigma^{\prime}}^{\perp}=\tau_{\rho \sigma ; \rho^{\prime} \sigma^{\prime}}^{\perp}, \tau_{\mu \nu ; \rho \sigma}^{\perp}=$ $\tau_{\rho \sigma ; \mu \nu}^{\perp}, \tau_{\mu \mu ; \rho \sigma}^{\perp}=0, \tau_{\mu \nu ; \mu \nu}^{\perp}=2$. The kinematical variables used here and below are introduced as $\omega \equiv \xi^{-1}=-P \cdot q / q^{2}$ (generalized Bjorken variable), $\eta=\Delta \cdot q / P \cdot q$ (skewedness), in terms of the vectors $P=P_{1}+P_{2}, q=\frac{1}{2}\left(q_{1}+q_{2}\right)$ and $\Delta=P_{2}-P_{1}=q_{1}-q_{2}$.

A simple calculation of one-loop diagrams (see Fig. 1) gives us the following result, with restriction of the reality of the final photon being relaxed, i.e. $q_{2}^{2} \neq 0$,

$$
T_{\mu \nu}=-\frac{\alpha_{s}}{\pi} T_{F} \sum_{i=1}^{N_{f}} Q_{i}^{2} \int_{-1}^{1} d t G_{\mu \nu}^{T}\left(t, \eta, \Delta^{2}\right) \sigma(t, \eta)\left\{1+\frac{1-\omega^{2} \eta^{2}}{\omega^{2}\left(t^{2}-\eta^{2}\right)} \ln \frac{1-t^{2} \omega^{2}}{1-\eta^{2} \omega^{2}}\right\}
$$

\footnotetext{
${ }^{2}$ The path-ordered link factor $\Phi\left[x_{2}, x_{1}\right]$ ensures gauge invariance.
} 
which agrees with Ref. [5]. The function $\sigma(t, \eta)$ appears from the conversion of gluon field (taken in the light-cone gauge) into strength tensor, $G_{+\mu}=\partial_{+} B_{\mu}$, and reads, for the fixing of the residual gauge symmetry consistent with canonical hamiltonian formalism [9,

$$
\sigma(t, \eta)=\frac{1}{(t-\eta+i 0)(t+\eta-i 0)} .
$$

Apart from (suppressed) momentum dependence of the coupling constant an additional source of scaling violation results from the renormalization of the composite operator (3) which will be discussed in the next two sections at one- and two-loop order.

\section{Evolution: anomalous dimensions.}

At leading order in the coupling constant the light-ray operator (3) obeys the light-cone position evolution equation of the form (here and below $\bar{y} \equiv 1-y$ )

$$
\frac{d}{d \ln \mu^{2}}\left[{ }^{G} \mathcal{O}^{T}\left(\kappa_{1}, \kappa_{2}\right)\right]=\frac{\alpha_{s}}{2 \pi} \int_{0}^{1} d z \int_{0}^{\bar{z}} d y^{G G} \mathcal{K}^{T}(y, z)\left[{ }^{G} \mathcal{O}^{T}\left(\bar{y} \kappa_{1}+y \kappa_{2}, z \kappa_{1}+\bar{z} \kappa_{2}\right)\right]
$$

with the kernel

$$
{ }^{G G} \mathcal{K}^{T}(y, z)=C_{A}\left\{y-2+\left[\frac{1}{y}\right]_{+}\right\} \delta(z)+C_{A}\left\{z-2+\left[\frac{1}{z}\right]_{+}\right\} \delta(y)-\frac{\beta_{0}}{2} \delta(y) \delta(z),
$$

where $\beta_{0}=\frac{4}{3} T_{F} N_{f}-\frac{11}{3} C_{A}$ is the first coefficient of the QCD $\beta$-function. Fourier transformation of this result gives the exclusive evolution kernel known before [10, 5, 11]. The tree level conformal invariance of the QCD Lagrangian allows to diagonalize this equation in the basis spanned by Gegenbauer polynomials?

$$
{ }^{G} \mathcal{O}_{j l}^{T}=G_{+\rho}\left(i \partial_{+}\right)^{l-1} \tau_{\mu \nu ; \rho \sigma}^{\perp} C_{j-1}^{5 / 2}\left(\overleftrightarrow{\mathcal{D}}_{+} / \partial_{+}\right) G_{\sigma+},
$$

which form an infinite dimensional representation of the conformal group in the space of bilinear operators. Therefore,

$$
\frac{d}{d \ln \mu^{2}}\left[{ }^{G} \mathcal{O}_{j l}^{T}\right]=-\frac{1}{2} \sum_{k=1}^{j}{ }^{G G} \gamma_{j k}^{T}\left[{ }^{G} \mathcal{O}_{j l}^{T}\right], \quad \text { with } \quad{ }^{G G} \gamma_{j}^{T(0)}=4 C_{A}(\psi(j+2)-\psi(1))+\beta_{0},
$$

the first term in the expansion ${ }^{G G} \gamma_{j k}^{T}=\left(\frac{\alpha_{s}}{2 \pi}\right){ }^{G G} \gamma_{j}^{T(0)} \delta_{j k}+\left(\frac{\alpha_{s}}{2 \pi}\right)^{2}{ }^{G G} \gamma_{j k}^{T(1)}+\mathcal{O}\left(\alpha_{s}^{3}\right)$.

In the momentum fraction space the evolution of the SPD can be done making use of orthogonal polynomial reconstruction (in the following Gegenbauer polynomials, $C_{j}^{5 / 2}$ ) of the function from its conformal moments according to Ref. 13.

$$
G\left(t, \eta, Q^{2}\right)=\sum_{j=1}^{N_{\max }} \widetilde{C}_{j-1}^{5 / 2}(t) \sum_{k=1}^{j} c_{j k}(\eta)\left(\frac{\alpha_{s}\left(Q_{0}^{2}\right)}{\alpha_{s}\left(Q^{2}\right)}\right)^{G G_{\gamma}^{T(0)} / \beta_{0}} \eta^{k-1} \int_{-1}^{1} d t C_{k-1}^{5 / 2}(t / \eta) G\left(t, \eta, Q_{0}^{2}\right),
$$

\footnotetext{
${ }^{3}$ We drop in what follows the Lorentz indices on the operators. Here $\partial=\vec{\partial}+\overleftarrow{\partial}$ and $\overleftrightarrow{\mathcal{D}}=\overrightarrow{\mathcal{D}}-\overleftarrow{\mathcal{D}}$
} 

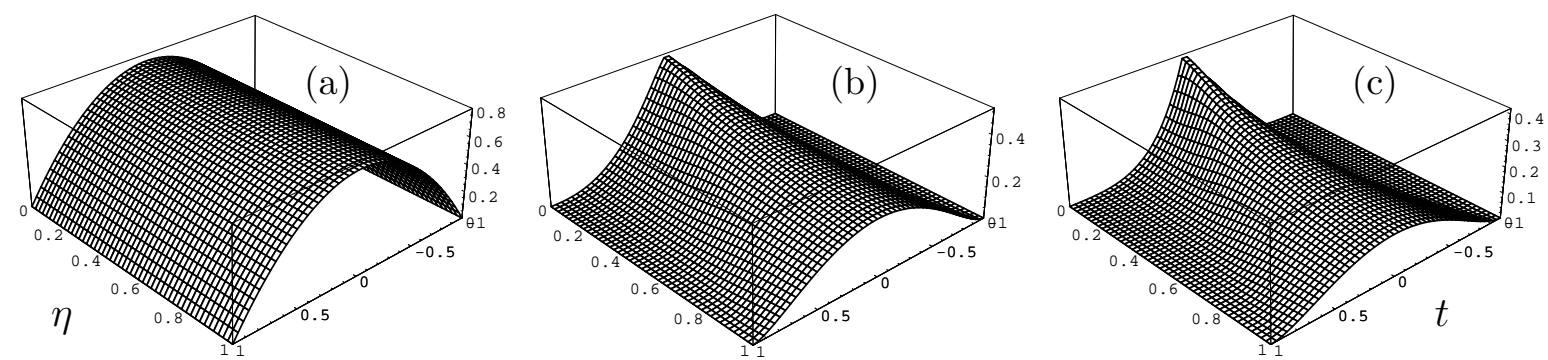

Figure 2: Input SPD in (a) and its evolved shape at $4 \mathrm{GeV}^{2}$ in (b) and at $100 \mathrm{GeV}^{2}$ in (c) for $N_{\max }=100$.

where formally $N_{\max }=\infty$. Here $\widetilde{C}_{j-1}^{5 / 2}(t)=\frac{9}{2} \frac{(2 j+3)}{(j)_{4}}\left(1-t^{2}\right)^{2} C_{j-1}^{5 / 2}(t)$ are adjoint polynomials and the re-expansion coefficients $c_{j k}(\eta)=\left\langle\widetilde{C}_{k-1}^{5 / 2}(t) \mid C_{j-1}^{5 / 2}(\eta t)\right\rangle$ are expressed in terms of hypergeometric function ${ }_{2} F_{1}\left(\eta^{2}\right)$. The evolution is demonstrated in Fig. 2 where we have taken an $\eta$-independent input' $G(t, \eta)=\frac{3}{4}\left(1-t^{2}\right)$, at very low $Q_{0}^{2}=0.2 \mathrm{GeV}^{2}$ and evolved it up to $Q^{2}=4 \mathrm{GeV}^{2}$ (b) and $Q^{2}=100 \mathrm{GeV}^{2}(\mathrm{c})$.

As a side remark on the solution of the leading order equation for "transversity" let us note that even in the case when any arbitrary number of gluons with the same helicity, whose pairwise interaction is described by the kernel (8), are exchanged in the $t$-channel, one can still diagonalize the (LO) multi-particle kernel since the problem admits a large enough number of conservation laws to be completely integrable. This can be found by noticing the equivalence of the anomalous dimensions (10) (which depend on the eigenvalues of $S L(2)$ Casimir operator $\hat{\boldsymbol{J}}^{2}$ ) to the Hamiltonian of the exactly solvable one-dimensional $X X X_{s=-3 / 2}$ spin chain model [11].

Next we compute the two-loop anomalous dimensions for the tensor gluon operator. To this end we use our machinery developed in Ref. [12]. Let us give a brief outline of the method. The structure of anomalous dimensions of conformal operators at two-loop (and higher) order reads

$$
{ }^{G G} \gamma_{j k}^{T(1)}={ }^{G G} \gamma_{j}^{T(1), \mathrm{D}} \delta_{j k}+{ }^{G G} \gamma_{j k}^{T(1), \mathrm{ND}}
$$

where ${ }^{G G} \gamma_{j}^{T(1), \mathrm{D}}$ are the next-to-leading order (NLO) forward anomalous dimensions [14]

$$
\begin{aligned}
{ }^{G G} \gamma_{j}^{T(1), \mathrm{D}} & =C_{A}^{2}\left\{S_{1}(j+1)\left(\frac{134}{9}-4 S_{2}^{\prime}\left(\frac{j+1}{2}\right)\right)-S_{3}^{\prime}\left(\frac{j+1}{2}\right)+8 \tilde{S}(j+1)-\frac{1}{j(j+3)}-\frac{16}{3}\right\} \\
& +C_{A} T_{F} N_{f}\left\{\frac{8}{3}-\frac{40}{9} S_{1}(j+1)-\frac{2}{j(j+3)}\right\}+C_{F} T_{F} N_{f} \frac{2(j+1)(j+2)}{j(j+3)}
\end{aligned}
$$

\footnotetext{
${ }^{4}$ We factored out the $\Delta^{2}$ dependence into the gluonic form factor $F_{G}\left(\Delta^{2}\right)$ which for phenomenological estimations can be taken equal $\kappa_{T}\left(1-\Delta^{2} / M_{\Lambda}^{2}\right)^{-3}$ with $M_{\Lambda}=2.7 \mathrm{GeV}$ and unknown parameter $\kappa_{T}$ which defines the magnitude of proton matrix element of the tensor gluonic operator.

${ }^{5}$ We thank W. Vogelsang for providing us his result for local anomalous dimensions.
} 


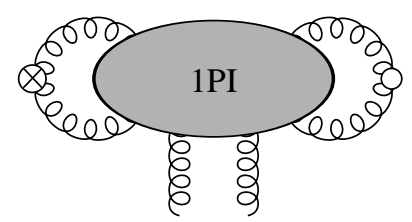

Figure 3: 1 PI Green function for calculation of the renormalization constant $Z_{A}^{-}$.

with

$$
S_{\ell}(j)=\sum_{k=1}^{j} \frac{1}{k^{\ell}}, \quad S_{\ell}^{\prime}\left(\frac{j}{2}\right)=2^{\ell} \sum_{k=1}^{j} \frac{\sigma_{k}}{k^{\ell}}, \quad \widetilde{S}(j)=\sum_{k=1}^{j} \frac{(-1)^{k}}{k^{2}} S_{1}(k),
$$

and where $\sigma_{j}=\frac{1}{2}\left[1+(-1)^{j}\right]$. The non-diagonal elements of anomalous dimension matrix of the conformal operators arise due to one-loop breaking of the conformal symmetry and, since the tree level conformal invariance leads to diagonal anomalous dimensions, the one-loop special conformal anomaly generates two-loop anomalous dimensions. The use of four-dimensional conformal algebra provides a relation between the anomalies of dilatation (read anomalous dimensions in question) and special conformal transformations via the commutator $\left[\mathcal{D}, \mathcal{K}_{-}\right]=i \mathcal{K}_{-}$which is applied on the Green function of elementary fields with conformal operator insertion. To evaluate the commutator the knowledge of scale and special conformal Ward identities, with unraveled pattern of symmetry breaking for afore mentioned Green function, is indispensable. A careful analysis reveals the result 12

$$
{ }^{G G} \gamma_{j k}^{T(1), \mathrm{ND}}=\left({ }^{G G} \gamma_{j}^{T(0)}-{ }^{G G} \gamma_{k}^{T(0)}\right)\left\{d_{j k}\left(\beta_{0}-{ }^{G G} \gamma_{k}^{T(0)}\right)+{ }^{G G} g_{j k}^{T}\right\}
$$

where ${ }^{G G} \gamma_{k}^{T(0)}$ are already known LO anomalous dimensions (10), $d_{j k}=-\sigma_{j-k}(2 k+3) /(j-k)$. $(j+k+3)$ for $j>k$ and $g_{j k}^{T}$ appears as a counterterm required for renormalization of the product of two composite operators: integrated anomaly $\mathcal{O}_{A}^{-} \equiv \int d^{4} x 2 x_{-} \mathcal{O}_{A}(x)=\int d^{4} x x_{-} Z_{3}\left(G_{\mu \nu}\right)^{2}$ in the trace of energy-momentum tensor and a conformal operator. The structure of the counterterms has been established using the form of counterterms for differential vertex operator insertions and found to be

$$
\begin{aligned}
i\left[\mathcal{O}_{A}(x)\right]\left[{ }^{G} \mathcal{O}_{j l}^{T}\right]=i\left[\mathcal{O}_{A}(x){ }^{G} \mathcal{O}_{j l}^{T}\right] & -\delta^{(d)}(x) \sum_{k=0}^{j}\left\{\hat{Z}_{A}\right\}_{j k}\left[{ }^{G} \mathcal{O}_{k l}^{T}\right]-\frac{i}{2} \partial_{+} \delta^{(d)}(x) \sum_{k=0}^{j}\left\{\hat{Z}_{A}^{-}\right\}_{j k}\left[{ }^{G} \mathcal{O}_{k l-1}^{T}\right]-\ldots \\
& -\left(g \frac{\partial \ln X}{\partial g}-2 \xi \frac{\partial \ln X}{\partial \xi}\right) B_{\mu}^{a}(x) \frac{\delta}{\delta B_{\mu}^{a}(x)}\left[{ }^{G} \mathcal{O}_{j l}^{T}\right]
\end{aligned}
$$

where $X=Z_{g} Z_{3}^{1 / 2}$ is expressed in terms of the renormalization constants of the gluon wave function $Z_{3}$ and the coupling $Z_{g}$.

To determine the unknown renormalization matrix $Z_{A}^{-}$it proves convenient to work in the lightcone position formalism and calculate 1PI Green function $\left\langle\left[\mathcal{O}_{A}(x)\right]\left[{ }^{G} \mathcal{O}^{T}\left(\kappa_{1}, \kappa_{2}\right)\right] B_{\mu}\left(x_{1}\right) B_{\nu}\left(x_{2}\right)\right\rangle_{\mathrm{amp}}$ 
shown in Fig. 3 (explicit one-loop graphs can be found in Ref. [12). We write the divergent part of the operator product $i\left[\mathcal{O}_{A}^{-}\right]\left[{ }^{G} \mathcal{O}^{T}\left(\kappa_{1}, \kappa_{2}\right)\right]$ via the following relation in leading order of the coupling constant:

$$
\begin{aligned}
i\left[\mathcal{O}_{A}^{-}\right]\left[{ }^{G} \mathcal{O}^{T}\left(\kappa_{1}, \kappa_{2}\right)\right] & =\frac{\alpha_{s}}{2 \pi} \frac{i}{\epsilon} \int_{0}^{1} d z \int_{0}^{\bar{z}} d y\left\{G G \mathcal{K}_{A}^{-}(y, z)\left[{ }^{G} \mathcal{O}^{T}\left(\bar{y} \kappa_{1}+y \kappa_{2}, z \kappa_{1}+\bar{z} \kappa_{2}\right)\right]\right. \\
& \left.+{ }^{G G} \widetilde{\mathcal{K}}_{A}^{-}(y, z) \int d^{d} x 2 x_{-} B_{\mu}^{b}(x) \frac{\delta}{\delta B_{\mu}^{b}(x)}\left[{ }^{G} \mathcal{O}^{T}\left(\bar{y} \kappa_{1}+y \kappa_{2}, z \kappa_{1}+\bar{z} \kappa_{2}\right)\right]\right\}
\end{aligned}
$$

For the evolution kernel $G G \widetilde{\mathcal{K}}_{A}^{-}$we find immediately from Eq. (15) that $G G \widetilde{\mathcal{K}}_{A}^{-}(y, z)=-i \frac{3}{4} \delta(y) \delta(z)$. Explicit calculation of the amputated Green function of conformal anomaly and conformal operator gives

$$
{ }^{G G} \mathcal{K}_{A}^{-}(y, z)=-i 2\left(\kappa_{1}+\kappa_{2}\right){ }^{G G} \mathcal{K}^{T}(y, z)-i\left(\kappa_{1}+\kappa_{2}\right) \beta_{0} \delta(y) \delta(z)+{ }^{G G} \mathcal{K}_{w}^{T}(y, z),
$$

where ${ }^{G G} \mathcal{K}^{T}(y, z)$ is the LO evolution kernel (8) and

$$
\begin{aligned}
{ }^{G G} \mathcal{K}_{w}^{T}(y, z) & =2 \frac{C_{A}}{k_{2+}}\left\{\left[\frac{2}{z}\right]_{+} \delta(y)-\left[\frac{1}{z^{2}}\right]_{+} \delta(y)+(2 y-1) \delta(z)\right\} \\
& +2 \frac{C_{A}}{k_{1+}}\left\{\left[\frac{2}{y}\right]_{+} \delta(z)-\left[\frac{1}{y^{2}}\right]_{+} \delta(z)+(2 z-1) \delta(y)\right\} .
\end{aligned}
$$

The evaluation of the conformal moments of (18) [multiplied by $1 / 2 \cdot(j-k) \cdot(j+k+3)$ ] gives us

$$
\begin{aligned}
{ }^{G G} g_{j k}^{T} & =-2 C_{A} \sigma_{j-k} \theta_{j-2, k} \frac{(3+2 k)}{(j-k)(j+k+3)} \\
& \times\left\{2 A_{j k}+\left(A_{j k}-\psi(j+2)+\psi(1)\right)\left[\frac{\Gamma(j+4) \Gamma(k)}{\Gamma(j) \Gamma(k+4)}-1\right]\right\},
\end{aligned}
$$

where we have introduced the matrix $A$ with its elements defined by

$$
A_{j k}=\psi\left(\frac{j+k+4}{2}\right)-\psi\left(\frac{j-k}{2}\right)+2 \psi(j-k)-\psi(j+2)-\psi(1),
$$

and $\theta_{j k}=1$ for $j>k$ and zero otherwise. Note that this result differs from our previous ones for chiral even operators only by a pure rational function

$$
{ }^{G G} g_{j k}^{T}={ }^{G G} g_{j k}^{V}+4 C_{A} \sigma_{j-k}(3+2 k) \frac{\Gamma(k)}{\Gamma(k+4)} .
$$

The solution of two-loop evolution equation is straightforward and can be found in Ref. [13].

\section{Evolution: two-loop kernel.}

In this section we give our results for the two-loop exclusive evolution kernel reconstructed from the known non-diagonal anomalous dimensions found above and two-loop splitting function of 
Ref. [14]. The structure of ER-BL kernel to $\mathcal{O}\left(\alpha_{s}^{3}\right)$ accuracy reads

$$
{ }^{G G} V^{T}=\frac{\alpha_{s}}{2 \pi}\left\{\left[{ }^{G G} V^{T(0)}\right]_{+}-\frac{1}{2}^{G G} \gamma_{1}^{T(0)} \delta(x-y)\right\}+\left(\frac{\alpha_{s}}{2 \pi}\right)^{2}\left\{\left[{ }^{G G} V^{T(1)}\right]_{+}-\frac{1}{2}^{G G} \gamma_{1}^{T(1)} \delta(x-y)\right\}
$$

where the +-prescription is defined according to

$$
[V(x, y)]_{+}=V(x, y)-\delta(x-y) \int_{0}^{1} d z V(z, y)
$$

Note that this prescription is not in one-to-one correspondence with the +-prescription in the forward case. The LO kernel reads [10, 5, 11]

$$
{ }^{G G} V^{T(0)}=C_{A} \theta(y-x)^{G G} f^{T}(x, y)+\left\{\begin{array}{l}
x \rightarrow \bar{x} \\
y \rightarrow \bar{y}
\end{array}\right\} \quad \text { with } \quad{ }^{G G} f^{T}=\frac{x^{2}}{y^{2}} \frac{1}{y-x} .
$$

To obtain the NLO correction we use the approach described in [15. Since the tensor gluon sector is almost analogous to handle as the vector and axial ones, we only mention the differences to these cases. The structure of two-loop anomalous dimensions (14) implies the following form of the kernel

$$
{ }^{G G} V^{T(1)}=-{ }^{G G} \dot{V}^{T} \otimes\left({ }^{G G} V^{T(0)}+\frac{\beta_{0}}{2}\right)-\left[G G g^{T} \otimes{ }^{G G} V^{T(0)}\right]-\frac{C_{A}^{2}}{2}{ }^{G G} G^{T}+{ }^{G G} D^{T},
$$

where the commutator stands for $[A \otimes, B](x, y)=\int_{0}^{1} d z\{A(x, z) B(z, y)-B(x, z) A(z, y)\}$. The off-diagonal part is contained in the first two convolutions on the r.h.s. of this equation and they are known exactly. The conformal moments of the dotted kernel,

$$
{ }^{G G} \dot{V}^{T(0)}=C_{A} \theta(y-x) \frac{x^{2}}{y^{2}} \frac{1}{y-x} \ln \frac{x}{y}+\left\{\begin{array}{l}
x \rightarrow \bar{x} \\
y \rightarrow \bar{y}
\end{array}\right\}
$$

are proportional to the commutator of the $d$-matrix with the LO anomalous dimensions. The $g$-matrix corresponds to the kernel

$$
{ }^{G G} g^{T}=C_{A} \theta(y-x)\left[2 \frac{x}{y}-\frac{\ln \left(1-\frac{x}{y}\right)}{y-x}\right]+\left\{\begin{array}{l}
x \rightarrow \bar{x} \\
y \rightarrow \bar{y}
\end{array}\right\}
$$

which contains a part (second term) of the chiral even (odd) case. The difference is a rational function (first term) which can be restored from the $G Q$-channel result by applying appropriate convolutions in order to trade the denominator $1 /(j+1) \cdot(j+2)$ of the $G Q$ conformal moments of $x / y$ to go over into the $G G$ sector , i.e. $1 / j \cdot(j+1) \cdot(j+2) \cdot(j+3)$.

\footnotetext{
${ }^{6}$ This can be achieved by convolution with the so-called $c$-kernel 15 in $Q Q$ channels and differentiation with respect to $y$, i.e. $d^{3} y^{2} \bar{y}^{2} / d y^{3}$. Finally, we removed a pure diagonal piece.
} 
Now the problem is reduced to the reconstruction of diagonal parts. The third term reads

$$
\begin{aligned}
{ }^{G G} G^{T} & =\left\{\theta(y-x)\left[{ }^{G G} h^{T}(x, y)+\Delta^{G G} h^{T}(x, y)\right]+\theta(y-\bar{x})\left[{ }^{G G} \bar{h}^{T}(x, y)+\Delta^{G G} h^{T}(\bar{x}, y)\right]\right\} \\
& +\left\{\begin{array}{l}
x \rightarrow \bar{x} \\
y \rightarrow \bar{y}
\end{array}\right\},
\end{aligned}
$$

and arises from the crossed-ladder diagram and can be obtained by means of $\mathcal{N}=1$ supersymmetry from the one in the quark sector. The particular contributions are

$$
\begin{aligned}
& { }^{G G} h^{T}=2^{G G} \bar{f}^{T} \ln \bar{x} \ln y-2{ }^{G G} f^{T}\left[\operatorname{Li}_{2}(x)+\operatorname{Li}_{2}(\bar{y})\right], \quad \Delta^{G G} h^{T}=-\frac{2 x}{y^{2} \bar{y}}-\frac{2 \bar{x}}{y^{2} \bar{y}} \ln \bar{x}-\frac{2 x}{y \bar{y}^{2}} \ln y, \\
& { }^{G G} h^{T}=\left({ }^{G G} f^{T}-{ }^{G G} \bar{f}^{T}\right)\left[2 \operatorname{Li}_{2}\left(1-\frac{x}{y}\right)+\ln ^{2} y\right]+2^{G G} f^{T}\left[\operatorname{Li}_{2}(\bar{y})-\ln x \ln y\right]+2^{G G} \bar{f}^{T} \operatorname{Li}_{2}(\bar{x}) .
\end{aligned}
$$

Here $\operatorname{Li}_{2}(x)=-\int_{0}^{x} \frac{d t}{t} \ln (1-t)$ is the Euler dilogarithm and we used the following shorthand notation ${ }^{G G} \bar{f}^{T}={ }^{G G} f^{T}(\bar{x}, \bar{y})$. The remaining diagonal part $D$ has a simple representation in terms of LO kernels and can be obtained by taking the forward limit and comparison with the known DGLAP kernel [14]. Consequent restoration of diagonal ER-BL kernels from the splitting functions is straightforward and gives

$$
\begin{aligned}
{ }^{G G} D^{T}= & -C_{F} T_{F} N_{f}\left[{ }^{G G} v^{a}+\frac{2}{3} G G v^{c}\right]+\beta_{0} C_{A}\left[\frac{3}{8} G G v^{a}-\frac{5}{6} G G v^{b}+\frac{1}{4} G G v^{c}\right] \\
& +C_{A}^{2}\left[\frac{13}{8} G G v^{a}-\frac{11}{6} G G v^{b}+\frac{13}{12} G G v^{c}\right] .
\end{aligned}
$$

Here the $b$ kernel coincides (with colour factor being dropped) with the LO kernel (24) and the $a$ and $c$ kernels having the structure $v^{i}(x, y)=f^{i} \theta(y-x)+\bar{f}^{i} \theta(x-y)$ are defined by the functions ${ }^{G G} f^{a}=x^{2} / y^{2}$ and ${ }^{G G} f^{c}=x^{2}(2 \bar{x} y+y-x) / y^{2}$. Eqs. (25) 30) is our final result for the NLO corrections to the gluon "transversity" evolution kernel.

\section{Cross sections.}

Now we are in a position to study the cross section for electroproduction of real photon where the gluonic SPD, whose perturbative properties we studied in detail in the previous sections, can be measured. For DVCS the skewedness $\eta$ and generalized Bjorken variable $\xi$ are proportional $\eta=-\xi\left(1+\frac{\Delta^{2}}{2 \mathcal{Q}^{2}}\right)^{-1}$. The differential cross section with unpolarized lepton beam and unpolarized target, in variables $y=P_{1} \cdot q_{1} / P_{1} \cdot k, \mathcal{Q}^{2} \equiv-q_{1}^{2}$ and $x \equiv \mathcal{Q}^{2} /\left(2 P_{1} \cdot q_{1}\right)$ with $\xi=x\left(1+\frac{\Delta^{2}}{2 \mathcal{Q}^{2}}\right)\left(2-x+x \frac{\Delta^{2}}{\mathcal{Q}^{2}}\right)^{-1}$, reads

$$
\frac{d \sigma}{d x d y d\left|\Delta^{2}\right| d \phi_{r}}=\frac{\alpha^{3} x y}{8 \pi \mathcal{Q}^{2}}\left(1+\frac{4 M^{2} x}{\mathcal{Q}^{2}}\right)^{-1 / 2}\left|\frac{\mathcal{T}}{e^{3}}\right|^{2} .
$$


Here $\phi_{r}$ is the azimuthal angle between the lepton and proton scattering planes in the rest frame of the target. In the following we will be interested only in the interference term $|\mathcal{I}|^{2} \equiv \mathcal{T}_{\mathrm{BH}} \mathcal{T}_{\mathrm{DVCS}}^{*}+$ $\mathcal{T}_{\text {DVCS }} \mathcal{T}_{\mathrm{BH}}^{*}$ between the DVCS, $\mathcal{T}_{\text {DVCS }}$, and Bethe-Heitler amplitude $\mathcal{T}_{\mathrm{BH}}$ since it provides a unique opportunity to extract the real/imaginary part of the gluonic amplitudes

$$
\left\{\begin{array}{c}
\mathcal{H}_{G}^{T}\left(\xi, \Delta^{2}\right) \\
\mathcal{E}_{G}^{T}\left(\xi, \Delta^{2}\right)
\end{array}\right\}=-\frac{\alpha_{s}}{\pi} T_{F} \sum_{i=1}^{N_{f}} Q_{i}^{2} \int_{-1}^{1} d t \sigma(t,-\xi)\left\{\begin{array}{c}
H_{G}^{T}\left(t, \xi, \Delta^{2}\right) \\
E_{G}^{T}\left(t, \xi, \Delta^{2}\right)
\end{array}\right\}
$$

An explicit calculation gives for unpolarized settings

$$
\begin{aligned}
\left|\frac{\mathcal{I}}{e^{3}}\right|^{2}=\frac{( \pm 1)}{2 \mathcal{Q}^{2} \Delta^{2}} \operatorname{Sp}\left\{\not k\left[\gamma_{\gamma}(\not k-\not \Delta)^{-1} \gamma_{\mu}+\gamma_{\mu}\left(\not k^{\prime}+\not \Delta\right)^{-1} \gamma_{\gamma}\right] \not k^{\prime} \gamma_{\nu}\right\} & \\
& \times \tau_{\mu \nu ; \alpha \beta}^{\perp} \Delta_{\alpha}\left\{\left(\xi g_{\beta \gamma}+\frac{\Delta_{\beta} q_{\gamma}}{P \cdot q}\right) \mathcal{T}_{1}+P_{\gamma} \Delta_{\beta} \mathcal{T}_{2}\right\}
\end{aligned}
$$

where

$$
\mathcal{T}_{1}=2\left(F_{1}+F_{2}\right) \operatorname{Re}\left(\mathcal{H}_{G}^{T}+\frac{\Delta^{2}}{4 M^{2}} \mathcal{E}_{G}^{T}\right), \quad \mathcal{T}_{2}=\frac{1}{2 M^{2}} \operatorname{Re}\left(F_{1} \mathcal{E}_{G}^{T}-F_{2} \mathcal{H}_{G}^{T}\right)
$$

and $+(-)$ sign stands for electron (positron) beam. In consequent evaluation of this expression we perform an expansion in $1 / \mathcal{Q}^{2}$ and keep only the first non-vanishing contribution. We form the charge asymmetry in order to extract the interference term from the total cross section. Since the double helicity flip amplitude presently considered is uniquely proportional to $\cos \left(3 \phi_{r}\right)$ (while all other contributions to the non-polarized cross section $|\mathcal{I}|^{2}$ enter with $\cos \left(n \phi_{r}\right)$ and $n=1,2$ [4, 16] one can isolate this purely gluonic contributions from the effects of quark SPD by forming an appropriate weighted cross section $\int d \phi_{r} w\left(\phi_{r}\right) \sigma\left(\phi_{r}\right)$ [4. Namely, choosing $w\left(\phi_{r}\right)=\cos \left(3 \phi_{r}\right)$ we extract tensor gluon SPD from the unpolarized cross section,

$$
\frac{1}{2 \pi} \int_{0}^{2 \pi} d \phi_{r} \cos \left(3 \phi_{r}\right) \frac{d^{+} \sigma-d^{-} \sigma}{d \phi_{r}}=16 \sqrt{-\frac{\Delta^{2}}{\mathcal{Q}^{2}}} \frac{\sqrt{(1-x)^{3}(1-y)}}{x y(2-x)^{2}}\left(1-\frac{\Delta_{\min }^{2}}{\Delta^{2}}\right)^{3 / 2} \mathcal{T}_{2} d \mathcal{M}
$$

where $d \mathcal{M}=\frac{\alpha^{3} x y}{8 \pi \mathcal{Q}^{2}}\left(1+\frac{4 M^{2} x}{\mathcal{Q}^{2}}\right)^{-1 / 2} d x d y d\left|\Delta^{2}\right|$ and $\Delta_{\min }^{2}=-M^{2} x^{2} /\left(1-x+x M^{2} / \mathcal{Q}^{2}\right)$. Note that the correction to the structure function $\mathcal{T}_{2}$ is suppressed by $\mathcal{O}\left(\sqrt{-\Delta^{2} / \mathcal{Q}^{2}}\right)$, while the combination $\mathcal{T}_{1}$ is down by $\mathcal{O}\left(M^{2} / \sqrt{-\Delta^{2} \mathcal{Q}^{2}}\right)$ relative to $M^{2} \mathcal{T}_{2}$.

The imaginary part of the amplitudes $\mathcal{H}_{G}^{T}$ and $\mathcal{E}_{G}^{T}$ can be accessed by means of single spin asymmetry. The polarization of the lepton beam does not induce contribution of gluon "transversity" distributions into the cross section in agreement with 《4. However, once the proton beam is longitudinally polarized we can access this asymmetry due to the interference term since the latter has genuine $\sin \left(3 \phi_{r}\right)$ azimuthal angle dependence. Thus the SPD can be extracted via the following weighted cross section

$$
\frac{1}{2 \pi} \int_{0}^{2 \pi} d \phi_{r} \sin \left(3 \phi_{r}\right) \frac{d^{+} \sigma_{\rightarrow}-d^{+} \sigma_{\leftarrow}}{d \phi_{r}}=16 \sqrt{-\frac{\Delta^{2}}{\mathcal{Q}^{2}}} \frac{\sqrt{(1-x)^{3}(1-y)}}{x y(2-x)^{2}}\left(1-\frac{\Delta_{\text {min }}^{2}}{\Delta^{2}}\right)^{3 / 2} \tilde{\mathcal{T}} d \mathcal{M}
$$


where in $d^{+} \sigma_{\rightarrow}$ the proton is polarized along the positron beam and

$$
\widetilde{\mathcal{T}}=\frac{1}{2 M^{2}} \operatorname{Im} F_{2}\left(\mathcal{H}_{G}^{T}+\frac{x}{2} \mathcal{E}_{G}^{T}\right) .
$$

Let us stress that the results (35) and (36) are valid to $1 / \sqrt{\mathcal{Q}^{2}}$ accuracy and further expansion terms from other structure functions can mimic the $\cos (3 \phi) / \sin (3 \phi)$ behaviour and contaminate the double helicity flip cross sections.

\section{Conclusion.}

In this paper we have presented weighted real photon electroproduction cross sections which can be used as a probe for the magnitude of gluon content in the nucleon. The opportunity to extract the real and imaginary parts of these amplitudes is offered by the scattering of the unpolarized lepton beam on the unpolarized and longitudinally polarized nucleon targets, respectively. We have given as well the formulae for NLO coefficient function as well as two-loop anomalous dimensions of the conformal operators and momentum fraction exclusive kernels thus completing the set of results required for study of the scaling violation for all twist-two SPDs at NLO.

This work was supported by DFG and BMBF (D.M.).

\section{References}

[1] R.L. Jaffe, A. Manohar, Phys. Lett. B 223 (1989) 218.

[2] A. Schäfer, L. Szymanowski, O.V. Teryaev, Phys. Lett. B 464 (1999) 94.

[3] N. Kivel, L. Mankiewicz, M.V. Polyakov, Phys. Lett. B 467 (1999) 263.

[4] M. Diehl, T. Gousset, B. Pire, J.P. Ralston, Phys. Lett. B 411 (1997) 193.

[5] P. Hoodbhoy, X. Ji, Phys. Rev. D 58 (1998) 054006.

[6] D. Müller, D. Robaschik, B. Geyer, F.-M. Dittes, J. Hořejši, Fortschr. Phys. 42 (1994) 101.

[7] X. Ji, Phys. Rev. Lett. 78 (1997) 610; Phys. Rev. D 55 (1997) 7114; J. Phys. G 24 (1998) 1181.

[8] A.V. Radyushkin, Phys. Lett. B 380 (1996) 417; Phys. Rev. D 56 (1997) 5524.

[9] A. Basseto, G. Nardelli, R. Soldati, Yang-Mills theories in the algebraic non-covariant gauges, World Scientific, (Singapore, 1991). 
[10] A.P. Bukhvostov, G.V. Frolov, L.N. Lipatov, E.A. Kuraev, Nucl. Phys. B 258 (1985) 601.

[11] A.V. Belitsky, Nucl. Phys. B 574 (2000) 407, hep-ph/9907420.

[12] D. Müller, Phys. Rev. D 49 (1994) 2525;

A.V. Belitsky, D. Müller, Nucl. Phys. B 537 (1999) 397.

[13] A.V. Belitsky, B. Geyer, D. Müller, A. Schäfer, Phys. Lett. B 421 (1998) 312;

A.V. Belitsky, D. Müller, L. Niedermeier, A. Schäfer, Phys. Lett. B 437 (1998) 160; Nucl. Phys. B 546 (1999) 279.

[14] W. Vogelsang, Acta Phys. Polon. B 29 (1998) 1189.

[15] A.V. Belitsky, D. Müller, A. Freund, Phys. Lett. B 461 (1999) 270; Phys. Lett. B 464 (1999) 249; Nucl. Phys. B (2000) (in press), hep-ph/9912379.

[16] A.V. Belitsky, D. Müller, L. Niedermeier, A. Schäfer, Leading twist asymmetries in deeply virtual Compton scattering, hep-ph/0004059. 